\title{
Geometry and Ergodic Theory of Parabolic Meromorphic Functions
}

\author{
Zuxing Xuan ${ }^{1}$
}

Received: 28 November 2021 / Accepted: 15 February 2022 / Published online: 5 March 2022

(c) The Author(s) 2022

\begin{abstract}
Let $f$ be a parabolic transcendental meromorphic function with positive and finite order $\rho$ and its derivative satisfies some growth conditions. In this paper, we show the existence of conformal measures and use this basic tool to illustrate both geometrical and dynamical features of the radial Julia set. We also characterize the conformal measures supported on radial Julia sets.
\end{abstract}

Keywords Parabolic meromorphic function · Perron-Frobenius operator · Pressure functions $\cdot$ Conformal measures

Mathematics Subject Classification Primary 30B20 $\cdot$ 30D05 $\cdot$ 30D10 $\cdot$ Secondary 34M05 - 37F10

\section{Introduction and Statement of Main Results}

As an important branch of complex dynamic systems, the measurable dynamics of rational functions is well-developed and has aroused many mathematicians' interests to work on it. This branch mainly focuses on the conformal measure and other measures and several types of dimensions of the Julia set. The concept of conformal measures is introduced by Patterson [1] as he investigated Fuchsian groups. Later, Sullivan [2] generalized it to the case of Kleinian groups. Sullivan [3, 4] gave the definition of conformal measures of rational functions. Now it has been the main tool to investigate the fractal geometry(including measures and dimensions) of the Julia set of rational functions. By using a parallel construction performed in the case of Kleinian groups, Sullivan [4] showed that for every rational function $f$ there exists at least one conformal measure. But his construction does not explicitly indicate the exponent of conformal measure. In order to get such information, a general scheme

Zuxing Xuan

zuxingxuan@163.com

1 Institute of Fundamental and Interdisciplinary Sciences, Beijing Union University,

Beijing 100101, China 
of constructing the conformal measures was proposed by Denker and Urbański in [5]. They had given many sufficient conditions to ensure the existence of conformal measures. For some special rational functions, conformal measures have good geometrical properties. These function classes include the following categories: hyperbolic rational functions, parabolic rational functions, geometrically finite rational functions, NCP(non-recurrent) functions, Collet-Eckmann functions, topological Collet-Eckmann functions, Fibonacci functions, etc.(for the related work we refer to [4, 6-23]).

In the middle of the 1990s, Barański [24] investigated the existence of conformal and invariant measures for transcendental meromorphic functions. But the further study encounters some difficulties due to lack of compactness, infinite degree of the map and more complicated geometry. In particular, topological entropy is usually infinite and the Ruelle inequality does not exist for general transcendental meromorphic functions. Researchers did not achieve remarkable results in this direction until the year of 2000(see [25-34], or refer to [35]). It is worth pointing out here that the above works are for some special (especially, periodic) transcendental meromorphic functions(e.g. exponential function family $\lambda e^{z}$ and elliptic functions and the functions with the form $H\left(e^{Q}\right)$ for rational functions $H$ and $Q$ ). One of the main thoughts has the dynamics of $f$ projected down onto the appropriate quotient space, either torus or infinite cylinder so that a lift $F$ of $f$ over the quotient space was obtained. Then the Perron-Frobenius-Ruelle operator can be defined for $F$. By adopting the operator theory, the conformal measures and invariant measures for $F$ were found. Then they projected the map $F$ onto $f$ to get some information about $f$. And a question arises: Can one investigate $f$ directly instead of using $F$ ? In order to solve the problem, Mayer and Urbański [36] introduced for the first time the following conditions.

Rapid growth condition There are $\alpha_{2}>\max \left\{0,-\alpha_{1}\right\}$ and $\kappa>0$ such that

$$
\left|f^{\prime}(z)\right| \geq \kappa^{-1}|z|^{\alpha_{1}}|f(z)|^{\alpha_{2}}
$$

for all $z \in J(f) \backslash\left(f^{-1}(\infty) \cup\{\infty\}\right)$.

Balanced growth condition There are $\alpha_{2}>\max \left\{0,-\alpha_{1}\right\}$ and $\kappa>0$ such that

$$
\kappa^{-1}|z|^{\alpha_{1}}|f(z)|^{\alpha_{2}} \leq\left|f^{\prime}(z)\right| \leq \kappa|z|^{\alpha_{1}}|f(z)|^{\alpha_{2}}
$$

for any $z \in J(f) \backslash\left(f^{-1}(\infty) \cup\{\infty\}\right)$.

Throughout this paper we use the notation $\alpha=\alpha_{1}+\alpha_{2}>0$. On the basis of (1.1) and (1.2), Mayer and Urbański adopted Nevanlinna's theory and the Riemannian metric $d \sigma=|z|^{-\alpha_{2}}|d z|$ to make the thermodynamic formalism work for a wide class of hyperbolic meromorphic functions of finite order. In particular, the existence of conformal(Gibbs) measures was established and then the existence of probability invariant measures equivalent to conformal measures was proved (see [36, 37]). Following this direction, Mayer and Urbański [38] further investigated ergodic properties of semi-hyperbolic functions with polynomial Schwarzian derivative.

A definition of hyperbolic meromorphic functions on the Riemann sphere is given in [39] and the thermodynamical formalism for these functions was considered in view of results of Walters as Kotus and Urbański [25] did. Later, the thermodynamical 
formalism for parabolic transcendental meromorphic function on the Riemann sphere (see Sect. 2 for the definition) was treated with in [40] by using a generalized version of Walters' results.

In this paper, we will explore the thermodynamical formalism of a class of nonhyperbolic transcendental meromorphic functions (we call them parabolic transcendental meromorphic functions on the complex plane, see Sect. 2 for the definition). The Julia sets of these functions contain finitely many rational indifferent periodic points. We must overcome the difficulties caused by these points.

One of our main results is the following.

Theorem 1.1 If $f: \mathbb{C} \rightarrow \widehat{\mathbb{C}}$ is a dynamically parabolic meromorphic function, then there exists a unique $h>\frac{\rho}{\alpha}$ such that $P(h)=0$ and $f$ has an $\left|f^{\prime}\right|_{\sigma}^{h}$-conformal measure $m_{h}$. Let $\mathcal{H}_{\sigma}^{t}(*)$ denote the $t$-dimensional Hausdorff measure of the set $*$ by means of the metric $d \sigma .\left.\mathcal{H}_{\sigma}^{h}\right|_{J_{r}(f)}$ is absolutely continuous with respect to $m_{h}$, where $J_{r}(f)=J^{*}(f) \backslash \bigcup_{n \geq 0} f^{-n}(\Omega)$. Moreover, the Radon-Nikodym derivative is bounded. $\operatorname{dim}_{H}\left(J_{r}(f)\right) \leq h$. If $\operatorname{dim}_{H}(I(f)) \leq \operatorname{dim}_{H}(J(f))$, then $\operatorname{dim}_{H}(J(f)) \leq h$.

The following theorem characterizes the $e^{P(t)}\left|f^{\prime}(z)\right|_{\sigma}^{t}$-conformal measures on Julia set of $f(z)$.

Theorem 1.2 Let $f(z)$ be a dynamically parabolic meromorphic function. Then for any fixed $t>\frac{\rho}{\alpha}$, two $e^{P(t)}\left|f^{\prime}\right|_{\sigma}^{t}$-conformal measures $m_{t}$ and $v_{t}$ are equivalent on $J_{r}(f)$ and the Radon-Nikodym derivative is bounded.

All the notations, such as $P(t), J^{*}(f)$ and the conformal measures, in Theorem 1.1 and Theorem 1.2 will be defined in below sections.

The paper consists of five sections. In Sect. 2, we collect a number of results that will be used later and establish some notations and definitions. Section 3 provides the definitions of the pressure function $P(t)$ and $e^{P(t)}\left|f^{\prime}(z)\right|_{\sigma}^{t}$-conformal measures. In section 4 , two lemmas are introduced. They come from analysis theory and play an crucial role in proving the existence of conformal measures. In Sect. 5, we give the proofs of Theorems 1.1 and 1.2.

In this paper, we use the following conventions to describe the relationship between two positive numbers $A$ and $B$. $A \asymp B$ means $A / C \leq B \leq C A$ for some implicit constant $C$. $A \preceq B$ means $A \leq C B$ for some implicit constant $C$.

\section{The Class of Functions and Definitions}

In this section, we first give the definition of some notations and then collect a number of results that will be used later in the paper.

Let $f(z)$ be a meromorphic function which is transcendental. Consider the $n$-th iterate of $f(z)$ defined by

$$
f^{0}(z)=z, f^{n}(z)=f\left(f^{n-1}(z)\right)=f^{n-1}(f(z)) .
$$


It is clear that if $f(z)$ is transcendental, that is, $\infty$ is an essential singular point of $f(z)$, then $f^{n}(z)$ is meromorphic only on $\mathbb{C} \backslash \cup_{j=1}^{n-1} f^{-j}(\infty)$.

The Fatou set $F(f)$ of a meromorphic function $f: \mathbb{C} \rightarrow \widehat{\mathbb{C}}$ is defined exactly in the same manner as for rational functions; $F(f)$ is the set of points $z \in \mathbb{C}$ such that all the iterates are defined and form a normal family on a neighborhood of $z$. The Julia set $J(f)$ is the complement of $F(f)$ in $\widehat{\mathbb{C}}$. Thus, $F(f)$ is open, $J(f)$ is closed, $F(f)$ is completely invariant while $f^{-1}(J(f)) \subseteq J(f)$ and $f(J(f) \backslash\{\infty\}) \subseteq J(f)$.

By Sing $\left(f^{-1}\right)$ we mean that the closure of the set of all finite critical and asymptotic values of $f(z)$ in the complex pane $\mathbb{C}$ and by $\widehat{\operatorname{Sing}}\left(f^{-1}\right)$ the closure of the set of all critical and asymptotic values of $f(z)$ in $\widehat{\mathbb{C}}=\mathbb{C} \cup\{\infty\}$. Hence if $f(z)$ has multiple poles, then $\infty$ is a critical value of $f(z)$ and $\infty \in \widehat{\operatorname{Sing}}\left(f^{-1}\right)$. If $\infty$ is an asymptotic value of $f(z)$, then $\infty \in \widehat{\operatorname{Sing}}\left(f^{-1}\right)$, but in any case, $\infty \notin \operatorname{Sing}\left(f^{-1}\right)$. Then $\infty \notin \widehat{\operatorname{Sing}}\left(f^{-1}\right)$ if and only if $f(z)$ has no multiple poles and no $\infty$ as an asymptotic value and $\infty$ is not a limit point of finite singular values of $f(z)$. We denote by $P(f)$ the postsingular set defined as the closure in $\widehat{\mathbb{C}}$ of

$$
\bigcup_{n=0}^{\infty} f^{n}\left(\operatorname{Sing}\left(f^{-1}\right) \backslash \bigcup_{j=0}^{n-1} f^{-j}(\infty)\right)
$$

and set $\widehat{P}(f)=P(f) \cup \widehat{\operatorname{Sin} g}\left(f^{-1}\right)$ and $\Omega=\Omega(f)=P(f) \cap J(f)$ for a meromorphic function $f$. We have to pay attention to the set $\Omega(f)$ when we study the dynamical behavior of $f$. The Speiser class $\mathcal{S}$ consists of transcendental meromorphic functions for which the set of singular values $\operatorname{Sing}\left(f^{-1}\right)$ is finite. The Eremenko-Lyubich class $\mathcal{B}$ is composed of transcendental meromorphic functions for which $\operatorname{Sing}\left(f^{-1}\right)$ is bounded.

A point $z_{0}$ is called periodic if for some $p \geq 1, f^{p}\left(z_{0}\right)=z_{0}$ and the least $p$ for the equality is period of $z_{0}$. A periodic point $z_{0}$ of period $p$ is called attracting, indifferent or repelling if $\left|\left(f^{p}\right)^{\prime}\left(z_{0}\right)\right|<1,=1$ or $>1$. If $\left(f^{p}\right)^{\prime}\left(z_{0}\right)=e^{i \alpha \pi}$ with a rational number $\alpha$, we regard $z_{0}$ to be a rationally indifferent periodic point of $f$. A rationally indifferent periodic point of $f$ must be a limit point of the forward orbits of a singular value, so it is in the derived set of $P(f)$. For our purpose of this paper, we have to pay much attention on the behavior of the function in a small neighborhood of a rationally indifferent periodic point.

The following definition was introduced in [41].

Definition 2.1 Let $f(z)$ be a transcendental meromorphic function on $\mathbb{C}$.

(1) $f(z)$ is called parabolic on the complex plane if

$$
1 \leq \sharp(P(f) \cap J(f))<\infty
$$

and every point in $P(f) \cap J(f)$ is a rationally indifferent periodic point of $f$ and $\operatorname{Sing}\left(f^{-1}\right) \subset F(f)$;

(2) $f(z)$ is called parabolic on the Riemann sphere(or with respect to the spherical metric in order to emphasize the considered metric) if $f(z)$ is parabolic on the complex plane and $\infty \notin \widehat{P}(f)$. 
We denote by $\mathcal{P}(\mathbb{C})($ resp. $\mathcal{P}(\widehat{\mathbb{C}}))$ the set of all parabolic transcendental meromorphic functions on $\mathbb{C}($ resp. $\widehat{\mathbb{C}})$.

Remark 2.1 1. From the above definition, all transcendental entire functions are not in $\mathcal{P}(\widehat{\mathbb{C}})$ because of $\infty \in \widehat{\mathcal{P}}(f)$. Actually, $\infty$ is an asymptotic value for all transcendental entire functions.

2. The functions in $\mathcal{P}(\widehat{\mathbb{C}})$ have infinitely many poles and all the poles are simple.

3. For $f \in \mathcal{P}(\widehat{\mathbb{C}}), \infty \notin \widehat{\operatorname{Sing}}\left(f^{-1}\right)$ and therefore $\mathcal{P}(\widehat{\mathbb{C}}) \subsetneq \mathcal{P}(\mathbb{C}) \cap \mathcal{B}$ by noting that $\infty$ can be an isolated singular value for the functions in $\mathcal{P}(\mathbb{C})$.

4. An example which was treated by Urbański and Zdunik [42] is $f_{\frac{1}{e}}(z)=\frac{1}{e} e^{z}$. This function belongs to $\mathcal{P}(\mathbb{C}) . f_{\frac{1}{e}}(z)$ has a unique rationally indifferent fixed point 1 .

Remark 2.2 Mayer and Urbański [36, 37] discussed in detail the meaning of the exponents $\alpha_{1}$ and $\alpha_{2}$ coming from (1.1) and (1.2), and they gave many examples. We can get functions in $\mathcal{P}(\mathbb{C})$ by choosing some parameters in their examples.

We consider three different metrics on $\mathbb{C}$ : Euclidean metric $|d z| ; d \sigma(z)=|z|^{-\alpha_{2}}|d z|$; Spherical metric $d_{\infty}(z)=\frac{|d z|}{1+|z|^{2}}$. The ball centered at $a(\neq 0)$ with radius $r$ with respect to the above three metrics is denoted by $B(a, r), B_{\sigma}(a, r)$ and $B_{\infty}(a, r)$ respectively. A simple calculation reveals the following fact:

We have the following estimate

$$
\begin{aligned}
\int_{b}^{b+r} x^{-\alpha_{2}} d x & =\left.\frac{1}{1-\alpha_{2}} x^{1-\alpha_{2}}\right|_{b} ^{b+r}=\frac{1}{1-\alpha_{2}}\left[(b+r)^{1-\alpha_{2}}-b^{1-\alpha_{2}}\right] \\
& =\frac{1}{1-\alpha_{2}} b^{1-\alpha_{2}}\left[\left(1+\frac{r}{b}\right)^{1-\alpha_{2}}-1\right] \\
& =\frac{1}{1-\alpha_{2}} b^{1-\alpha_{2}}\left[1+\frac{r}{b}\left(1-\alpha_{2}\right)-1+o\left(\frac{r}{b}\right)\right] \asymp b^{-\alpha_{2}} r,
\end{aligned}
$$

where $\alpha_{2} \neq 1, b>0$ and

$$
\int_{b}^{b+r} x^{-1} d x=\left.(\ln x)\right|_{b} ^{b+r}=\ln \left(1+\frac{r}{b}\right) \asymp b^{-\alpha_{2}} r,
$$

where $\alpha_{2}=1, b>0$.

From above, the change of the metrics results in the change of the radius of balls: the ball $B(a, r)$ with $\left|\frac{r}{a}\right|<<1$ characterized by the metric $|d z|$ becomes approximately $B_{\sigma}\left(a,|a|^{-\alpha_{2}} r\right)$ with respect to the metric $d \sigma(z)$.

This fact will be frequently used in the following context. We can see that these two metrics are equivalent to each other on any bounded set with a positive distance from 0. In virtue of Definition 2.1, we know that the Julia set of these functions is not the whole complex plane. Therefore, we can assume that $0 \in F(f)$ (otherwise it suffices to conjugate the function by a translation). This is enough for the purpose of our study in considering the metric $d \sigma$ (otherwise, we have to use the metric $d \sigma=\left(1+|z|^{\alpha_{2}}\right)^{-1}|d z|$ instead) since in this case, 0 has a positive distance from $J(f)$. 
For $f(z) \in \mathcal{P}(\mathbb{C}), \Omega=\left\{\omega_{1}, \ldots, \omega_{k}\right\}$ consists of all rational indifferent periodic points with $1 \leq k<\infty$ and set $B(\Omega, \Theta):=\bigcup_{i=1}^{k} B\left(\omega_{i}, \Theta\right)$. The dynamical behavior in a neighborhood of a rationally indifferent periodic point was discussed in Denker and Urbański [43] in view of the Fatou's Flower Theorem.

Lemma 2.1 Let $f \in \mathcal{P}(\mathbb{C})$. For every $\Theta>0$ there exists $\delta=\delta(\Theta)>0$ such that for every $a \in J(f) \backslash B(\Omega, \Theta)$, we have $B(a, 2 \delta) \cap \mathcal{P}(f)=\emptyset$. In particular, all analytic branches of the inverse of $f^{n}$ are well defined on $B(a, 2 \delta)$ and $B(f(a), 2 \delta)$ for every $n=1,2, \ldots$.

Denker and Urbański [43] proved the following result for rational parabolic functions, we can get the similar results for transcendental meromorphic functions in $\mathcal{P}(\mathbb{C})$.

Lemma 2.2 Let $f \in \mathcal{P}(\mathbb{C})$. Then there exists $\Theta>0$ such that for $z \in J(f) \backslash \bigcup_{n \geq 0} f^{-n}(\Omega \cup\{\infty\}), f^{n}(z) \in J(f) \backslash B(\Omega, \Theta)$ for infinitely many $n$.

Remark 2.3 We will use the corresponding version of Lemmas 2.1 and 2.2 with the balls replaced by $B_{\sigma}(\cdot, \cdot)$. Put

$$
B_{\sigma}\left(\Omega, \Theta \cdot|\Omega|^{-\alpha_{2}}\right)=\bigcup_{i=1}^{k} B_{\sigma}\left(\omega_{i}, \Theta \cdot|\Omega|^{-\alpha_{2}}\right),
$$

where $|\Omega|^{-\alpha_{2}}:=\max _{1 \leq i \leq k}\left|\omega_{i}\right|^{-\alpha_{2}}$. Using the metric $d \sigma$ in Lemma 2.2, we know $f^{n_{j}}(z) \in J(f) \backslash B_{\sigma}\left(\Omega, \Theta \cdot|\Omega|^{-\alpha_{2}}\right)$ for an unbounded sequence $\left\{n_{j}\right\}$.

\section{Growth Conditions, Pressure, Perron-Frobenius-Ruelle Operators and Generalised Conformal Measures}

The basic concepts of thermodynamic formalism are the Perron-Frobenius-Ruelle operator and eigenvector of its dual operator, called in the sequel a generalised conformal measure.

The derivative of a function $f: \mathbb{C} \rightarrow \widehat{\mathbb{C}}$ with respect to the metric $d \sigma(z)=|z|^{-\alpha_{2}}|d z|$ is given at a point $z \in \mathbb{C}$ by the formula

$$
\left|f^{\prime}(z)\right|_{\sigma}=\frac{d \sigma(f(z))}{d \sigma(z)}=\left|f^{\prime}(z)\right| \frac{|z|^{\alpha_{2}}}{|f(z)|^{\alpha_{2}}} .
$$

Definition 3.1 ([36]) A meromorphic function $f$ is of divergent type if the series

$$
\Sigma(t, w)=\sum_{z \in f^{-1}(w)}|z|^{-t}
$$


diverges at the convergent exponent, i.e., at the order $t=\rho$ of the function for any value $w$ which is not Picard exceptional. In the case $f$ is entire we assume instead of (3.1) that, for any $A, B>0$, there exists $R>1$ such that

$$
\int_{\log R}^{R} \frac{T(r, f)}{r^{\rho+1}} d r-B(\log R)^{1-\rho} \geq A
$$

where $T(r, f)=m(r, f)+N(r, f)$ is the Nevanlinna characteristic of $f$,

$$
N(r, f)=\int_{0}^{r} \frac{n(t, f)-n(0, f)}{t} d t+n(0, f) \log r,
$$

$n(r, f)$ is the number of poles of $f(z)$ in $\{z:|z| \leq r\}$; for $x>0$, let $\log ^{+} x=\max \{\log x, 0\}$,

$$
m(r, f)=\frac{1}{2 \pi} \int_{0}^{r} \log ^{+}\left|f\left(r e^{\mathrm{i} \theta}\right)\right| d \theta .
$$

The order of $f(z)$ is defined as follows

$$
\rho(f)=\limsup _{r \rightarrow \infty} \frac{\log T(r, f)}{\log r} .
$$

We call $f(z)$ dynamically parabolic if $f \in \mathcal{P}(\mathbb{C})$ with positive and finite order $\rho$ satisfies the balanced derivative growth condition (1.2) and is of divergent type.

Koebe's distortion theorem gives the following distortion theorem with respect to the metric $d \sigma$.

Lemma 3.1 If $f \in \mathcal{P}(\mathbb{C})$ satisfies the rapid growth condition and suppose that $B_{\sigma}\left(w, 2 \delta|w|^{-\alpha_{2}}\right) \subset \mathbb{C} \backslash \mathcal{P}(f)$. Then for every $n \geq 1, z \in f^{-n}(w)$ and all $x, y \in B_{\sigma}\left(w, \delta|w|^{-\alpha_{2}}\right)$, we have that

$$
K_{\sigma}^{-1} \leq \frac{\left|\left(f_{z}^{-n}\right)^{\prime}(y)\right|_{\sigma}}{\left|\left(f_{z}^{-n}\right)^{\prime}(x)\right|_{\sigma}} \leq K_{\sigma}
$$

for some universal constant $K_{\sigma} \geq 1$.

Following Mayer and Urbański [36], we define Perron-Frobenius-Ruelle operator(with the potential $\left.\phi_{t}(z)=-t \log \left|f^{\prime}(z)\right|_{\sigma}\right)$ : For $w \in J(f) \backslash(\Omega \cup\{\infty\})$, define

$$
\mathcal{L}_{t}(\varphi)(w)=\sum_{z \in f^{-1}(w)}\left|f^{\prime}(z)\right|_{\sigma}^{-t} \varphi(z), \varphi \in \mathcal{C}_{b}(J(f) \backslash(\Omega \cup\{\infty\})) .
$$

By adopting a similar approach to Theorem 4.5 of [36] and noting that the exceptional set $\mathcal{E}_{f} \subset \operatorname{Sing}\left(f^{-1}\right) \subseteq F(f)$ in Definition 2.1, we can prove that: Given a parabolic meromorphic function $f$ of finite order satisfying the rapid growth condition, $\mathcal{L}_{t}\left(t>\frac{\rho}{\alpha}\right)$ acts continuously on the Banach space $\mathcal{C}_{b}(J(f) \backslash(\Omega \cup\{\infty\}))$ of bounded 
continuous functions on $J(f) \backslash(\Omega \cup\{\infty\})$. Therefore we can consider the iterate of the operator by calculating

$$
\mathcal{L}_{t}^{n}(\varphi)(w)=\mathcal{L}_{t}\left(\mathcal{L}_{t}^{n-1}(\varphi)\right)(w)=\sum_{f^{n}(z)=w} \varphi(z)\left|\left(f^{n}\right) \prime(z)\right|_{\sigma}^{-t} .
$$

Hence we have

$$
\mathcal{L}_{t}^{n}(\mathbf{1})(w)=\sum_{f^{n}(z)=w}\left|\left(f^{n}\right) \prime(z)\right|_{\sigma}^{-t}
$$

Based on this operator, we can define the topological pressure and conformal measure as follows.

Definition 3.2 The topological pressure of $f$ at $w \in J(f) \backslash \Omega \cup\{\infty\}$ for $t>\frac{\rho}{\alpha}$ is defined as follows:

$$
P_{w}(f, t)=\limsup _{n \rightarrow \infty} \frac{1}{n} \log \mathcal{L}_{t}^{n}(\mathbf{1})(w) .
$$

Definition 3.3 A probability measure $m_{t}$ is called $\lambda|f \prime(z)|_{\sigma}^{t}$-conformal with $\lambda>0$ if one of the following equivalent properties holds:

(1) For every measurable set $E \subset J(f)$ such that $\left.f\right|_{E}$ is injective we have

$$
m_{t}(f(E))=\int_{E} \lambda|f \prime(z)|_{\sigma}^{t} d m_{t} .
$$

(2) $m_{t}$ is an eigenmeasure of the adjoint $\mathcal{L}_{t}^{*}$ of the transfer operator $\mathcal{L}_{t}$ with eigenvalue $\lambda$ :

$$
\mathcal{L}_{t}^{*} m_{t}=\lambda m_{t}
$$

Remark 3.1 If in Definition 3.3 the conformal factor $\lambda=1$, the measure $m_{t}$ is simply called $t$-conformal. Usually, $\lambda=\exp P_{w}(f, t)$ for some $w \in J(f)$.

Zheng [41] investigated the pressure regarding the spherical metric for a parabolic function in $\mathcal{P}(\widehat{\mathbb{C}})$.

We can prove the following lemma by applying the same method as in [41].

Lemma 3.2 If $f \in \mathcal{P}(\mathbb{C})$ and satisfies the rapid growth condition. Then for any $a, b \in J(f) \backslash(\Omega \cup\{\infty\}), P_{a}(f, t)=P_{b}(f, t)$. We write $P(f, t)$ for $P_{a}(f, t)$ for $a \in J(f) \backslash(\Omega \cup\{\infty\})$. We simply write $P(t)$ when $f$ is clear.

Proof Assume that $P_{a}(f, t)<+\infty$. For arbitrarily small $\varepsilon>0$ and for all sufficiently large $n$, we have 


$$
\begin{aligned}
\exp \left\{n\left(P_{a}(f, t)+\varepsilon\right)\right\} & \geq \mathcal{L}_{t}^{n}(\mathbf{1})(a) \\
& =\sum_{f^{n}(z)=a} \frac{1}{\left|\left(f^{n}\right) \prime(z)\right|_{\sigma}^{t}} \\
& =\sum_{f^{p}(w)=a f^{n-p}(z)=w} \frac{1}{\left|\left(f^{p}\right) \prime(w)\right|_{\sigma}^{t}} \frac{1}{\left|\left(f^{n-p}\right) \prime(z)\right|_{\sigma}^{t}} \\
& \geq \frac{1}{\left|\left(f^{p}\right) \prime(w)\right|_{\sigma}^{t}} \sum_{f^{n-p}(z)=w} \frac{1}{\left|\left(f^{n-p}\right) \prime(z)\right|_{\sigma}^{t}},
\end{aligned}
$$

where $w \in f^{-p}(a)$. The remaining proof is the same as that of [41].

\section{Two Lemmas from Analysis Theory}

The following is the Besicovitch covering lemma(see Theorem 3.2.1 in [44]), which is of importance in discussing conformal measures.

Lemma 4.1 Let $A \subset \mathbb{R}^{n}$ (n-dimensional Euclidean space) be a bounded set, $r(x): A \rightarrow(0, \infty)$ is a function. Then there exists a countable subset $L$ of $A$ such that

$$
A \subset \bigcup_{x \in L} B(x, r(x))
$$

Meanwhile, every point in $A \subset \mathbb{R}^{n}$ is contained in at most $4^{2 n}$ balls $B(x, r(x))(x \in L)$, that is, the multiplicity of covering is no larger than $4^{2 n}$.

Remark 4.1 The lemma still holds when the metric is the spherical metric(or the metric $d \sigma$ ), but the constant $4^{4}$ in the lemma should be another constant $\chi(2)$ (or $\sigma(2))$ which is independent of the covering.

We will obtain the desired conformal measure through the limit measure of weak convergence of a sequence of pure atom probability measures. Therefore, the following Prokhorov's theorem is the key tool in achieving our purpose.

Lemma 4.2 (Prokhorov's theorem [44]) Let $\mathbf{P}\left(\mathbb{R}^{k}\right)$ be the collection of probability measures on $k$-dimensional Euclidean space $\mathbb{R}^{k}$. If $\left\{\mu_{n}\right\}$ is a tight sequence in $\mathbf{P}\left(\mathbb{R}^{k}\right)$, then there exist a subsequence $\left\{\mu_{n_{j}}\right\}$ and a probability measure $\mu \in \mathbf{P}\left(\mathbb{R}^{k}\right)$ such that $\left\{\mu_{n_{j}}\right\}$ converges weakly to $\mu$.

We recall the tightness of a probability measure family. A family $\mathcal{F}$ of Borel probability measures on metric space $(E, \mathfrak{B})$ is tight, if $\forall \varepsilon>0$, there exists a compact subset $K$ of $E$, such that $\forall \mu \in \mathcal{F}, \mu\left(K^{C}\right)<\varepsilon$, where $K^{C}=E \backslash K$. Therefore, for a compact metric space, any family of probability measures is tight and is relatively compact under weak topology. 


\section{Proofs of Theorems 1.1 and 1.2}

We first give the following lemma which tells us some information of the pressure function.

Lemma 5.1 If $f(z)$ is a dynamically parabolic meromorphic function, then for every $t>\frac{\rho}{\alpha}$, the following statements are true:

(1) The topological pressure $P(t)=\lim _{n \rightarrow \infty} \frac{1}{n} \log \mathcal{L}_{t}^{n}(\mathbf{1})(w)$ exists and is independent of $w \in J(f) \backslash(\Omega \cup\{\infty\}), P(t)$ is convex and consequently, continuous in $t$;

(2) The function $t \mapsto P(t)$ is strictly decreasing in $(\rho / \alpha,+\infty)$;

(3) $\lim _{t \rightarrow+\infty} P(t)=-\infty$;

(4) $\lim _{t \rightarrow(\rho / \alpha)+} P(t)>0$;

(5) there exists unique $h>\frac{\rho}{\alpha}$ such that $P(h)=0$, in fact $h=\inf \{t: P(t) \leq 0\}$.

Proof The proof of (1) and (4) are the same as the proof of Proposition 7.2 of [36]. For every $k \geq 1$, put

$$
X_{k}=\left(J(f) \backslash \bigcup_{n \geq 0} f^{-n}(\infty)\right) \backslash \bigcup_{n \geq 0} f^{-n}\left(B\left(\Omega, \frac{1}{k}\right)\right) .
$$

According to Theorem 2.4 of [39], we can prove $f$ is expanding on $X_{k}$ concerning the Euclidean metric, that is, for some positive integer $N$, some $\lambda>1$ and $\delta>0$,

$$
d\left(f^{N}(z), f^{N}(w)\right) \geq \lambda d(z, w),
$$

whenever $z$ and $w$ are in a common component of $f^{-N}(B(a, \delta))$ for some $a \in X_{k}$. From this we can obtain (2) and (3). (5) follows from (1), (2), (3) and (4).

The following lemma is a special case of Proposition 4.22 of [37] which gives the support of the conformal measure.

Lemma 5.2 Suppose that $m$ is an arbitrary $\lambda e^{-\phi}$-conformal measure with some $\lambda>0$. Then there exists $M>0$ such that for $m$-a.e. $x \in J(f)$, we have

$$
\liminf _{n \rightarrow \infty}\left|f^{n}(x)\right| \leq M
$$

Particularly, $m\left(I_{\infty}(f)\right)=0$, where $I_{\infty}(f)=I(f) \cup J_{\infty}=I(f) \bigcup\left(\cup_{n \geq 0} f^{-n}(\infty)\right)$.

Here $I(f)$ is the escaping set defined by $I(f)=\left\{z \in J(f): f^{n}(z) \rightarrow \infty(n \rightarrow \infty)\right\}$. We also need the following definition. 
Definition 5.1 [23] For a sequence $\left\{a_{n}: n \geq 1\right\}$ of the real numbers,

$$
c=\limsup _{n \rightarrow \infty} \frac{a_{n}}{n}
$$

will be called the transition parameter of $\left\{a_{n}: n \geq 1\right\}$.

The transition parameter is actually determined by the property that

$$
\sum_{n \geq 1} \exp \left(a_{n}-n s\right)
$$

converges for $s>c$ and diverges for $s<c$ and hence it is also called transition parameter for the series. We are in the position to give the proof of the Theorem 1.1.

Proof of Theorem 1.1 The proof consists of two steps.

Step 1 Firstly, we prove the existence of the $e^{P(t)}\left|f^{\prime}\right|_{\sigma}^{t}$-conformal measure for the parabolic meromorphic functions which are of positive and finite order and satisfy rapid growth condition by adopting ideas in [36, 37] with some modifications.

Fix $w \in J(f) \backslash(\Omega \cup\{\infty\})$. From the discussion in Section 3, we know that for $t>\frac{\rho}{\alpha}$, the transition parameter for the series

$$
\Sigma_{\tau}=\sum_{n=1}^{\infty} e^{-n \tau} \mathcal{L}_{t}^{n}(\mathbf{1})(w)
$$

is the topological pressure $P(t)$ (not depending on the choice of $w \in J(f) \backslash(\Omega \cup\{\infty\}))$. In other words, $\Sigma_{\tau}=+\infty$ for $\tau<P(t), \quad \Sigma_{\tau}<+\infty$ for $\tau>P(t)$. In order to make the discussion easy, here we assume that $\Sigma_{P(t)}=+\infty$, otherwise we can use the method of Denker and Urbański's [5] to modify the series suitably to achieve our purpose.

For $\tau>P(t)$, put

$$
\nu_{\tau}=\frac{1}{\Sigma_{\tau}} \sum_{n=1}^{\infty} e^{-n \tau}\left(\mathcal{L}_{t}^{n}\right)^{*} \delta_{w} .
$$

Then

$$
\begin{aligned}
\nu_{\tau}(\mathbb{C}) & =\int_{\mathbb{C}} \mathbf{1} d \nu_{\tau}=\int_{\mathbb{C}} \mathbf{1} d\left(\frac{1}{\Sigma_{\tau}} \sum_{n=1}^{\infty} e^{-n \tau}\left(\mathcal{L}_{t}^{n}\right)^{*} \delta_{w}\right) \\
& =\frac{1}{\Sigma_{\tau}} \sum_{n=1}^{\infty} e^{-n \tau}\left(\int_{\mathbb{C}} \mathbf{1} d\left(\mathcal{L}_{t}^{n}\right)^{*} \delta_{w}\right) \\
& =\frac{1}{\Sigma_{\tau}} \sum_{n=1}^{\infty} e^{-n \tau} \mathcal{L}_{t}^{n}(\mathbf{1})(w)=1 .
\end{aligned}
$$


This indicates that $\left(v_{\tau}\right)_{\tau>P(t)}$ is a set of probability measures on $\mathbb{C}$. In view of the noncompactness of the space $(\mathbb{C}, d \sigma)$, we will use Prokhorov's theorem to get the conformal measure. What we need to prove is that the family of measures is tight, that is to say, for any $\varepsilon>0$, there exists a constant $R>0$, such that for all $\tau>P(t)$, we have $v_{\tau}\left(W_{R}\right)<\varepsilon$, where $W_{R}=\{z \in \mathbb{C}:|z|>R\}$.

The rapid growth condition gives that

$$
\begin{aligned}
\mathcal{L}_{t}\left(\mathbf{1}_{W_{R}}\right)(w) & =\sum_{z \in f^{-1}(w) \cap W_{R}}\left|f^{\prime}(z)\right|_{\sigma}^{-t} \\
& \leq \kappa^{t} \sum_{z \in f^{-1}(w) \cap W_{R}}|z|^{-\alpha t} \\
& \leq \frac{\kappa^{t}}{R^{\frac{\alpha t-\rho}{2}}} \sum_{z \in f^{-1}(w)} \frac{1}{|z|^{\frac{\rho+\alpha t}{2}}} \leq \frac{\kappa^{t} M_{\frac{\rho+\alpha t}{2}}}{R^{\frac{\alpha t-\rho}{2}}}
\end{aligned}
$$

where $M_{\frac{\rho+\alpha t}{2}}$ is a constant. Therefore,

$$
\begin{aligned}
\mathcal{L}_{t}^{n+1}\left(\mathbf{1}_{W_{R}}\right)(w) & =\sum_{y \in f^{-n}(w)} \sum_{z \in f^{-1}(y) \cap W_{R}} \frac{1}{\left(\left|f^{\prime}(z)\right|_{\sigma}\left|\left(f^{n}\right)^{\prime}(y)\right|_{\sigma}\right)^{t}} \\
& =\sum_{y \in f^{-n}(w)} \frac{1}{\left|\left(f^{n}\right)^{\prime}(y)\right|_{\sigma}^{t}} \mathcal{L}_{t}\left(\mathbf{1}_{W_{R}}\right)(y) \\
& \leq \frac{\kappa^{t} M_{\frac{\rho+\alpha t}{2}}^{\frac{\alpha t-\rho}{2}}}{R_{t}^{\frac{\alpha t}{2}}(\mathbf{1})(w) .}
\end{aligned}
$$

Thus for every $\tau>P(t)$, we have

$$
\begin{aligned}
\nu_{\tau}\left(W_{R}\right) & =\frac{1}{\Sigma_{\tau}} \sum_{n=1}^{\infty} e^{-n \tau} \mathcal{L}_{t}^{n}\left(\mathbf{1}_{W_{R}}\right)(w) \\
& \leq \frac{\kappa^{t} M_{\frac{\rho+\alpha t}{2}}^{\frac{\alpha t-\rho}{2}}}{R^{\frac{1}{2}}} \sum_{n=1}^{\infty} e^{-n \tau} \mathcal{L}_{t}^{n-1}(\mathbf{1})(w) \\
& =\frac{\kappa^{t} M_{\frac{\rho+\alpha t}{2}}^{\frac{\alpha t-\rho}{2}}}{R_{\tau}} \frac{1}{\Sigma^{\tau}}\left[1+\sum_{n=1}^{\infty} e^{-n \tau} \mathcal{L}_{t}^{n}(\mathbf{1})(w)\right] \\
& \leq 2 \frac{\kappa^{t} M_{\frac{\rho+\alpha t}{2}}^{\frac{\alpha t-\rho}{2}}}{R^{\frac{\rho}{2}}} \frac{1}{e^{P(t)}}
\end{aligned}
$$

The tightness of the family $\left\{v_{\tau}\right\}_{\tau>P(t)}$ follows.

Now, choose a sequence $\left\{s_{j}\right\}_{j=1}^{\infty}, s_{j}>P(t)$ satisfying $\lim _{j \rightarrow \infty} s_{j}=P(t)$. In view of Prokhorov's theorem, passing to a subsequence, we may assume without loss of generality that the sequence $\left\{v_{s_{j}}\right\}_{j=1}^{\infty}$ converges weakly to a Borel probability measure $m_{t}$ on $J(f)$. From 


$$
\begin{aligned}
\frac{1}{e^{\tau}} \mathcal{L}_{t}^{*} \nu_{\tau} & =\frac{1}{\Sigma_{\tau}} \sum_{n=1}^{\infty} e^{-(n+1) \tau}\left(\mathcal{L}_{t}^{n+1}\right)^{*} \delta_{w} \\
& =v_{\tau}-\frac{1}{\Sigma_{\tau}} \frac{\mathcal{L}_{t}^{*} \delta_{w}}{e^{\tau}}
\end{aligned}
$$

and $\Sigma_{P(t)}=+\infty$, letting $s_{j} \rightarrow P(t)$ in the above equality, we get $\mathcal{L}_{t}^{*} m_{t}=e^{P(t)} m_{t}$. Thus $m_{t}$ is a conformal measure on $J(f)$. Combining Lemma 5.1 with Step 1 gives the proof of the first part of Theorem 1.1, that is to say, there exists a unique $h>\frac{\rho}{\alpha}$ such that $P(h)=0$ and $f$ has an $\left|f^{\prime}\right|_{\sigma}^{h}$-conformal measure $m_{h}$.

Step 2 We prove the remaining part of the theorem. Put

$$
\begin{gathered}
J_{M}^{*}(f)=\left\{z \in J(f): \liminf _{n \rightarrow \infty}\left|f^{n}(z)\right|<M\right\} \\
J^{*}(f)=\bigcup_{M>0} J_{M}^{*}(f)=J(f) \backslash I_{\infty}(f) .
\end{gathered}
$$

Lemma 5.2 implies that there exists $M>0$ such that

$$
m_{h}\left(J_{M}^{*}(f)\right)=1 .
$$

Fix $z \in J_{M}^{*}(f) \backslash\left(\bigcup_{n \geq 0} f^{-n}(\Omega \cup\{\infty\})\right)$. In view of Lemma 2.2 and Remark 2.3, there exists a sequence $n_{j}=n_{j}(z)(j=1,2, \ldots)$ of positive integers increasing to infinity such that $f^{n_{j}}(z) \in J_{M}^{*}(f) \backslash B_{\sigma}\left(\Omega, \Theta \cdot|\Omega|^{-\alpha_{2}}\right)$ for every $j=1,2, \ldots$ By Lemma 2.1 , for every $j=1,2, \ldots$, there exists a unique analytic inverse branch $f_{z}^{-n_{j}}: B_{\sigma}\left(f^{n_{j}}(z), 2 \delta\left|f^{n_{j}}(z)\right|^{-\alpha_{2}}\right) \rightarrow \mathbb{C}$ of $f^{n_{j}}$ such that $f_{z}^{-n_{j}}\left(f^{n_{j}}(z)\right)=z$. By Lemma 3.1, we deduce that

$$
f_{z}^{-n_{j}}\left(B_{\sigma}\left(f^{n_{j}}(z), \delta \cdot\left|f^{n_{j}}(z)\right|^{-\alpha_{2}}\right)\right) \supset B_{\sigma}\left(z, r_{j}|z|^{-\alpha_{2}}\right)
$$

and

$$
f^{n_{j}}\left(B_{\sigma}\left(z, r_{j}|z|^{-\alpha_{2}}\right)\right) \supset B_{\sigma}\left(f^{n_{j}}(z), K_{\sigma}^{-2} \delta\left|f^{n_{j}}(z)\right|^{-\alpha_{2}}\right),
$$

where

$$
r_{j}=r_{j}(z)=\frac{\left|\left(f_{z}^{-n_{j}}\right)^{\prime}\left(f^{n_{j}}(z)\right)\right|_{\sigma}}{K_{\sigma}} \delta=\frac{\delta}{K_{\sigma}\left|\left(f^{n_{j}}\right)^{\prime}(z)\right|_{\sigma}} \rightarrow 0 \text { as } j \rightarrow \infty .
$$

Since $J(f) \cap \overline{B(0, M)}$ is a compact set of $\mathbb{C}$ (with respect to the Euclidean metric and $d \sigma)$, we have

$$
R_{M}:=\inf \left\{m_{h}\left(B_{\sigma}\left(w, \delta|w|^{-\alpha_{2}}\right)\right): w \in J^{*}(f) \cap B(0, M)\right\}>0 .
$$

We write $n=n_{j}(z)$. By Lemma 3.1, we have 


$$
\begin{aligned}
B_{\sigma}\left(z, K_{\sigma}^{-1} \delta\left|f^{n}(z)\right|^{-\alpha_{2}}\left|\left(f^{n}\right)^{\prime}(z)\right|_{\sigma}^{-1}\right) & \subset f_{z}^{-n}\left(B_{\sigma}\left(f^{n}(z), \delta\left|f^{n}(z)\right|^{-\alpha_{2}}\right)\right) \\
& \subset B_{\sigma}\left(z, K_{\sigma} \delta\left|f^{n}(z)\right|^{-\alpha_{2}}\left|\left(f^{n}\right)^{\prime}(z)\right|_{\sigma}^{-1}\right) .
\end{aligned}
$$

Since $m_{h}$ is the $h$-conformal measure, we have

$$
\begin{aligned}
& m_{h}\left(f_{z}^{-n}\left(B_{\sigma}\left(f^{n}(z), \delta\left|f^{n}(z)\right|^{-\alpha_{2}}\right)\right)\right) \\
& \quad \asymp\left|\left(f^{n}\right)^{\prime}(z)\right|_{\sigma}^{-h} m_{h}\left(B_{\sigma}\left(f^{n}(z), \delta\left|f^{n}(z)\right|^{-\alpha_{2}}\right)\right) .
\end{aligned}
$$

It follows from (5.8) and (5.9) that

$$
\begin{aligned}
& m_{h}\left(B_{\sigma}\left(z, K_{\sigma} \delta\left|f^{n}(z)\right|^{-\alpha_{2}}\left|\left(f^{n}\right)^{\prime}(z)\right|_{\sigma}^{-1}\right)\right) \\
& \geq\left|\left(f^{n}\right)^{\prime}(z)\right|_{\sigma}^{-h} m_{h}\left(B_{\sigma}\left(f^{n}(z), \delta\left|f^{n}(z)\right|^{-\alpha_{2}}\right)\right) \\
& \geq R_{M}\left(K_{\sigma} \delta\right)^{-h} \Gamma^{\alpha_{2} h}\left(K_{\sigma} \delta\left|f^{n}(z)\right|^{-\alpha_{2}}\left|\left(f^{n}\right)^{\prime}(z)\right|_{\sigma}^{-1}\right)^{h} .
\end{aligned}
$$

That is

$$
\frac{m_{h}\left(B_{\sigma}\left(z, K_{\sigma} \delta\left|f^{n}(z)\right|^{-\alpha_{2}}\left|\left(f^{n}\right)^{\prime}(z)\right|_{\sigma}^{-1}\right)\right)}{\left(K_{\sigma} \delta\left|f^{n}(z)\right|^{-\alpha_{2}}\left|\left(f^{n}\right)^{\prime}(z)\right|_{\sigma}^{-1}\right)^{h}} \geq c R_{M}\left(K_{\sigma} \delta\right)^{-h} \Gamma^{\alpha_{2} h}>0 .
$$

Thus, there exists a constant $\eta>0$ such that, for every $z \in J_{M}^{*}(f) \backslash \bigcup_{n \geq 0} f^{-n}(\Omega)$,

$$
\limsup _{r \rightarrow 0} \frac{m_{h}\left(B_{\sigma}(z, r)\right)}{r^{h}} \geq \limsup _{n \rightarrow \infty} \frac{m_{h}\left(B_{\sigma}\left(z, K_{\sigma} \delta\left|f^{n}(z)\right|^{-\alpha_{2}}\left|\left(f^{n}\right)^{\prime}(z)\right|_{\sigma}^{-1}\right)\right)}{\left(K_{\sigma} \delta\left|f^{n}(z)\right|^{-\alpha_{2}}\left|\left(f^{n}\right)^{\prime}(z)\right|_{\sigma}^{-1}\right)^{h}} \geq \eta .
$$

This deduces that

$$
\mathcal{H}_{\sigma}^{h}\left(J_{M}^{*}(f) \backslash \bigcup_{n \geq 0} f^{-n}(\Omega)\right) \leq \eta^{-1} m_{h}\left(J_{M}^{*}(f) \backslash \bigcup_{n \geq 0} f^{-n}(\Omega)\right) \leq \eta^{-1} .
$$

We also have the following

$$
\begin{aligned}
\mathcal{H}_{\sigma}^{h}\left(J_{M+k+1}^{*}(f) \backslash J_{M+k}^{*}(f)\right) & \leq \eta^{-1} m_{h}\left(J_{M+k+1}^{*}(f) \backslash J_{M+k}^{*}(f)\right) \\
& =\eta^{-1}\left(m_{h}\left(J_{M+k+1}^{*}(f)\right)-m_{h}\left(J_{M+k}^{*}(f)\right)\right) \\
& =\eta^{-1}(1-1)=0 .
\end{aligned}
$$

It is easy to see that

$$
J^{*}(f)=J_{M}^{*}(f) \cup \bigcup_{k=0}^{\infty}\left(J_{M+k+1}^{*}(f) \backslash J_{M+k}^{*}(f)\right)
$$

Therefore, we have 


$$
\begin{aligned}
& \mathcal{H}_{\sigma}^{h}\left(J^{*}(f) \backslash \cup_{n \geq 0} f^{-n}(\Omega)\right) \\
= & \mathcal{H}_{\sigma}^{h}\left(\left(J_{M}^{*}(f) \backslash \cup_{n \geq 0} f^{-n}(\Omega)\right) \cup \bigcup_{k=0}^{\infty}\left(J_{M+k+1}^{*}(f) \backslash J_{M+k}^{*}(f)\right)\right) \\
= & \mathcal{H}_{\sigma}^{h}\left(J_{M}^{*}(f) \backslash \cup_{n \geq 0} f^{-n}(\Omega)\right)+\sum_{k=0}^{\infty} \mathcal{H}_{\sigma}^{h}\left(J_{M+k+1}^{*}(f) \backslash J_{M+k}^{*}(f)\right) \\
= & \mathcal{H}_{\sigma}^{h}\left(J_{M}^{*}(f) \backslash \cup_{n \geq 0} f^{-n}(\Omega)\right) \leq \eta^{-1} .
\end{aligned}
$$

This implies that $\operatorname{dim}_{H}\left(J_{r}(f)\right) \leq h$. The same argument yields the result that $\left.\mathcal{H}_{\sigma}^{h}\right|_{J_{r}(f)}$ is absolutely continuous with respect to $m_{h}$, and the Radon-Nikodym derivatives bounded from above.

Noticing that $\bigcup_{n \geq 0} f^{-n}(\Omega \cup\{\infty\})$ is countable, we have $\operatorname{dim}_{H}\left(\bigcup_{n \geq 0} f^{-n}(\Omega \cup\{\infty\})\right)$ $=0$. If $\operatorname{dim}_{H}\left(I_{\infty}(f)\right)<\operatorname{dim}_{H}(J(f))$, then

$$
\operatorname{dim}_{H}(J(f))=\operatorname{dim}_{H}\left(J(f) \backslash\left(I(f) \bigcup \cup_{n \geq 0} f^{-n}(\Omega \cup\{\infty\})\right) \leq h .\right.
$$

Remark 5.1 The conformal measures achieved by Mayer and Urbański [36, 37] depend on the choice of the starting point. For the hyperbolic case, the unique $\left|f^{\prime}\right|_{\sigma}^{t}$ -conformal measure got by Mayer and Urbański [36, 37] is non-atomic.

For $f \in \mathcal{P}(\mathbb{C}) \cap \mathcal{B}$, Zheng [41] proved that it has finitely many and at least one parabolic domain and at most finitely many attracting domains without other types of stable domains. This indicates there does not exist escaping point in $F(f)$, and so it is $I(f) \subseteq J(f)$. However, we can not prove that $\operatorname{dim}_{H}(J(f)) \leq h$ although we have $m_{h}\left(I_{\infty}(f)\right)=0$.

\subsection{Proof of Theorem 1.2}

Similarly to the proof of Theorem 1.1 and using some ideas of Mayer and Urbański [37], there is $M>0$ so large that $m_{t}\left(J_{M}^{*}(f)\right)=1$. Since $J(f) \cap \overline{B(0, M)}$ is a compact set of $\mathbb{C}$ (with respect to the Euclidean metric and $d \sigma$ ),

$$
R_{M}:=\inf \left\{m_{t}\left(B_{\sigma}\left(w, \delta K_{\sigma}^{-2}|w|^{-\alpha_{2}}\right)\right): w \in J^{*}(f) \cap B(0, M)\right\}>0 .
$$

Therefore,

$$
\begin{aligned}
1 & \geq m_{t}\left(f^{n_{j}}\left(B_{\sigma}\left(z, r_{j}|z|^{-\alpha_{2}}\right)\right)\right. \\
& =\int_{B_{\sigma}\left(z, r_{j}|z|^{-\alpha_{2}}\right)} e^{n_{j} P(t)}\left|\left(f^{n_{j}}\right)^{\prime}(w)\right|_{\sigma}^{t} d m_{t} \\
& \geq K_{\sigma}^{-t} e^{n_{j} P(t)}\left|\left(f^{n_{j}}\right)^{\prime}(z)\right|_{\sigma}^{t} m_{t}\left(B_{\sigma}\left(z, r_{j}|z|^{-\alpha_{2}}\right)\right) \\
& =1 \delta^{t} K_{\sigma}^{-2 t} r_{j}^{-t} e^{n_{j} P(t)} m_{t}\left(B_{\sigma}\left(z, r_{j}|z|^{-\alpha_{2}}\right)\right),
\end{aligned}
$$


1 where $r_{j}$ is defined as in (5.7), and

$$
\begin{aligned}
R_{M} & \leq m_{t}\left(B_{\sigma}\left(f^{n_{j}}(z), K_{\sigma}^{-2} \delta\left|f^{n_{j}}(z)\right|^{-\alpha_{2}}\right)\right. \\
& \leq m_{t}\left(f^{n_{j}}\left(B_{\sigma}\left(z, r_{j}|z|^{-\alpha_{2}}\right)\right)\right) \\
& =\int_{B_{\sigma}\left(z, r_{j}|z|^{\left.-\alpha_{2}\right)}\right.} e^{n_{j} P(t)}\left|\left(f^{n_{j}}\right)^{\prime}(w)\right|_{\sigma}^{t} d m_{t} \\
& \leq K_{\sigma}^{t}\left|\left(f^{n_{j}}\right)^{\prime}(z)\right|_{\sigma}^{t} e^{n_{j} P(t)} m_{t}\left(B_{\sigma}\left(z, r_{j}|z|^{-\alpha_{2}}\right)\right) \\
& =\delta^{t} r_{j}^{-t} e^{n_{j} P(t)} m_{t}\left(B_{\sigma}\left(z, r_{j}|z|^{-\alpha_{2}}\right)\right) .
\end{aligned}
$$

So

$$
R_{M} \delta^{-t} \leq \frac{m_{t}\left(B_{\sigma}\left(z, r_{j}|z|^{-\alpha_{2}}\right)\right)}{e^{-n_{j} P(t)} r_{j}^{t}} \leq K_{\sigma}^{2 t} \delta^{-t}
$$

Thus there exists some constant $B_{M}\left(m_{t}\right) \geq 1$ depending on $m_{t}$ and $M$,

$$
B_{M}\left(m_{t}\right)^{-1} \leq \frac{m_{t}\left(B_{\sigma}\left(z, r_{j}|z|^{-\alpha_{2}}\right)\right)}{e^{-n_{j} P(t)} r_{j}^{t}} \leq B_{M}\left(m_{t}\right) .
$$

Suppose that $v_{t}$ is a $e^{P(t)}\left|f^{\prime}\right|_{\sigma}^{t}$-conformal measure. Then $v_{t}\left(I_{\infty}(f)\right)=0$. From the above we know that there exists $M>0$ such that $v_{t}\left(J_{M}^{*}(f)\right)=m_{t}\left(J_{M}^{*}(f)\right)=1$.

Fix $z \in J_{M}^{*}(f) \backslash \bigcup_{n=0}^{\infty} f^{-n}(\Omega)$. Then there exist $y \in J(f) \cap B(0, M)$ and an increasing sequence $\left\{n_{k}\right\}_{k=1}^{\infty}$ such that $y=\lim _{k \rightarrow \infty} f^{n_{k}}(z)$. For a certain $k$ large enough, considering the sets $f_{z}^{-n_{k}}\left(B_{\sigma}\left(y, 2 \delta|y|^{-\alpha_{2}}\right)\right)$ and $f_{z}^{-n_{k}}\left(B_{\sigma}\left(y, \delta|y|^{-\alpha_{2}} /\left(2 K_{\sigma}\right)\right)\right)$, where $f_{z}^{-n_{k}}$ is the holomorphic inverse branch of $f^{n_{k}}$ defined on $B_{\sigma}\left(y, 4 \delta|y|^{-\alpha_{2}}\right)$ and sending $f^{n_{k}}(z)$ to $z$. For all $k \geq 1$ large enough, we have

$$
\begin{aligned}
B_{M}\left(v_{t}\right)^{-1} e^{-n_{k} P(t)}\left|\left(f^{n_{k}}\right) \prime(z)\right|_{\sigma}^{t} & \leq v_{t}\left(B_{\sigma}\left(z, \frac{\delta}{2}|z|^{-\alpha_{2}}\left|\left(f^{n_{k}}\right)^{\prime}(z)\right|^{-1}\right)\right) \\
& \leq B_{M}\left(v_{t}\right) e^{-n_{k} P(t)}\left|\left(f^{n_{k}}\right) \prime(z)\right|_{\sigma}^{t} .
\end{aligned}
$$

Fix now $E$, an arbitrary bounded Borel set contained in $J^{*}(f)$ and let $E_{M}=E \cap J_{M}^{*}(f)$. Since $v_{t}$ is regular, for every $z \in E_{M}$ there exists a radius $r(z) \in(0, \varepsilon)$ such that

$$
v_{t}\left(\left(\bigcup_{x \in E_{M}} B_{\sigma}(x, r(x))\right) \backslash E_{M}\right) \leq \varepsilon .
$$

According to the Besicovi $\breve{c}$ Covering Theorem, we can choose a countable subcover $\left\{B_{\sigma}\left(z_{i}, r\left(z_{i}\right)\right)\right\}_{i=1}^{\infty}$ with $r\left(z_{i}\right) \leq \varepsilon$ and $n\left(z_{i}\right) \geq \varepsilon^{-1}$, from the cover $\left\{B_{\sigma}(z, r(z))\right\}_{z \in E^{\prime}}$ of $E^{\prime}$, of multiplicity bounded by some constant $C \geq 1$, independent of the cover. Therefore, 


$$
\begin{aligned}
m_{t}(E) & =m_{t}\left(E_{M}\right) \leq \sum_{i=1}^{\infty} m_{t}\left(B_{\sigma}\left(z_{i}, r\left(z_{i}\right)\right)\right) \\
& \leq B_{M}\left(m_{t}\right) \sum_{i=1}^{\infty} e^{-n\left(z_{i}\right) P(t)}\left|\left(f^{n\left(z_{i}\right)}\right) \prime\left(z_{i}\right)\right|_{\sigma}^{t} \\
& \leq B_{M}\left(m_{t}\right) B_{M}\left(v_{t}\right) \sum_{i=1}^{\infty} v_{t}\left(B_{\sigma}\left(z_{i}, r\left(z_{i}\right)\right)\right) \\
& \leq B_{M}\left(m_{t}\right) B_{M}\left(v_{t}\right) \cdot \sigma(2) \cdot v_{t}\left(\bigcup_{i=1}^{\infty} B_{\sigma}\left(z_{i}, r\left(z_{i}\right)\right)\right) \\
& \leq \sigma(2) B_{M}\left(m_{t}\right) B_{M}\left(v_{t}\right) v_{t}\left(\bigcup_{i=1}^{\infty} B_{\sigma}\left(z_{i}, r\left(z_{i}\right)\right)\right) \\
& \leq \sigma(2) B_{M}\left(m_{t}\right) B_{M}\left(v_{t}\right)\left(\varepsilon+m_{t}\left(E_{M}\right)\right) \\
& =\sigma(2) B_{M}\left(m_{t}\right) B_{M}\left(v_{t}\right)\left(\varepsilon+m_{t}(E)\right) .
\end{aligned}
$$

Letting $\varepsilon \searrow 0$, we obtain $v_{t}(E) \leq \sigma(2) B_{M}\left(m_{t}\right) B_{M}\left(m_{t}\right) m_{t}(E)$. Exchanging $m_{t}$ with $v_{t}$, we obtain $m_{t}(E) \leq \sigma(2) B_{M}\left(m_{t}\right) B_{M}\left(v_{t}\right) v_{t}(E)$, which implies that the measures $m_{t}$ and $v_{t}$ are equivalent on $J_{r}(f)=J^{*}(f) \backslash \bigcup_{n \geq 0} f^{-n}(\Omega)$ with Radon-Nikodym derivatives bounded away from zero and infinity.

In the end, we give a conjecture.

Conjecture 5.1 For the functions $f(z)$ in Theorem 1.1, Bowen formula follows

$$
\operatorname{dim}_{H}\left(J_{r}(f)\right)=h,
$$

where $h$ is the unique zero of the pressure function $P(t)$.

Remark 5.2 From Theorem 1.1 we know that the crucial step to prove 5.1 is the proof of $\operatorname{dim}_{H}\left(J_{r}(f)\right) \geq h$. For the hyperbolic case, Mayer and Urbański [36, 37] used $f$-invariance and the ergodic measure equivalent to $m_{h}$.

Remark 5.3 The existence of $f$-invariant measure plays an important role in the measurable dynamic theory. The results of Walters' and Martens's research are frequently used to find the desired measures. Here we conjecture that for the dynamically parabolic meromorphic function, there exists $f$-invariant measure equivalent to $e^{P(t)}\left|f^{\prime}(z)\right|_{\sigma}^{t}$-conformal measure $m_{t}$ (when it is atomless).

\section{Conclusions}

For a parabolic transcendental meromorphic function $f$ with positive and finite order $\rho$. Given some growth conditions on its derivative, we show the existence of conformal measures and use this basic tool to illustrate both geometrical and dynamical 
features of the radial Julia set. These results are meaningful to further reveal the dynamic behavior of meromorphic functions.

Acknowledgements The author is supported in part by Beijing outstanding talents training fund youth top individual project (Grant no. 2018000026833ZK57) and Premium Funding Project for Academic Human Resources Development in Beijing Union University (Grant no. BPHR2020EZ01).

Author Contributions ZX is the only contributor in preparing the manuscript.

Funding Beijing outstanding talents training fund youth top individual project (Grant no. 2018000026833ZK57) and Premium Funding Project for Academic Human Resources Development in Beijing Union University (Grant no. BPHR2020EZ01).

Availability of data and material All data generated or analysed during this study are included in this published article.

\section{Declarations}

Conflict of interests The author declares that he has no competing interests.

Ethics approval and consent to participate Not applicable.

Consent for publication Not applicable.

Open Access This article is licensed under a Creative Commons Attribution 4.0 International License, which permits use, sharing, adaptation, distribution and reproduction in any medium or format, as long as you give appropriate credit to the original author(s) and the source, provide a link to the Creative Commons licence, and indicate if changes were made. The images or other third party material in this article are included in the article's Creative Commons licence, unless indicated otherwise in a credit line to the material. If material is not included in the article's Creative Commons licence and your intended use is not permitted by statutory regulation or exceeds the permitted use, you will need to obtain permission directly from the copyright holder. To view a copy of this licence, visit http://creativecommons.org/licen ses/by/4.0/.

\section{References}

1. Patterson, S.J.: The limit set of a Fuchsian group. Acta Math. 136(3-4), 241-273 (1976)

2. Sullivan, D.: The density at infinity of a discrete group. Inst. Hautes Etudes Sci. Publ. Math. 50, 171-202 (1979)

3. Sullivan, D.: Seminar on conformal and hyperbolic geometry. Preprint IHES (1982)

4. Sullivan, D.: Conformal dynamical systems. In: Siva, S. (ed.) Geometric Dynamics, Lecture Notes in Mathematics, vol. 1007, pp. 725-752. Springer, Berlin (1983)

5. Denker, M., Urbański, M.: On the existence of conformal measures. Trans. Am. Math. Soc. 328(2), 563-587 (1991)

6. Denker, M., Urbański, M.: On Sullivan's conformal measures for rational maps of the Riemann sphere. Nonlinearity 4(2), 365-384 (1991)

7. Denker, M., Urbański, M.: The capacity of parabolic Julia sets. Math. Z. 211(1), 73-86 (1992)

8. Denker, M., Urbański, M.: Geometric measures for parabolic rational maps. Ergodic. Theory Dyn. Syst. 12(1), 53-66 (1992)

9. Denker, M., Urbański, M.: On Hausdorff measures on Julia sets of subexpanding rational maps. Israel J. Math. 76, 193-214 (1991) 
10. Denker, M., Urbański, M.: Ergodic theory of equilibrium states for rational maps. Nonlinearity 4, 103-134 (1991)

11. Graczyk, J., Smirnov, S.: Non-uniform hyperbolicity in complex dynamics. Invent. Math. 175(2), 335-415 (2009)

12. McMullen, C.T.: Hausdorff dimension and conformal dynamics. II. Geometrically finite rational maps. Comment. Math. Helv. 75(4), 535-593 (2000)

13. Stratmann, B.O., Urbański, M.: The geometry of conformal measures for parabolic rational maps. Math. Proc. Camb. Philos. Soc. 128, 141-156 (2000)

14. Urbański, M.: On Hausdorff dimension of a Julia set with a rationally periodic point. Studia Math. 97(3), 167-188 (1991)

15. Urbański, M.: Rational functions with no recurrent critical points. Ergodic Theory Dyn. Syst. 14(2), 391-414 (1994)

16. Urbański, M.: Geometry and ergodic theory of conformal nonrecurrent dynamics. Ergodic Theory Dyn. Syst. 17(6), 1449-1476 (1997)

17. Urbański, M.: Measures and dimensions in conformal dynamics. Bull. Am. Math. Soc. 40(3), 281321 (2003)

18. Havard, G.: Mesures invariantes pour les fractions rationnelles geometriquement finies. Fund. Math. 160(1), 39-61 (1999)

19. Przytycki, F.: Iterations of holomorphic Collet-Eckmann maps: conformal and invariant measures. Trans. Am. Math. Soc. 350(2), 717-742 (1998)

20. Przytycki, F.: On measure and Hausdorff dimensions of Julia sets for holomorphic Collet-Eckmann maps. In: International conference on dynamical systems, Montevideo. Pitman Research Notes of Mathematics, vol. 362, pp. 167-181 (1995)

21. Przytycki, F., Rivera-Letelier, J.: Statistical properties of topological Collet-Eckmann maps. Ann. Sci. Econ. Norm. Sup. 40, 135-178 (1995)

22. Przytycki, F., Urbański, M.: Porosity of Julia sets of non-recurrent and parabolic Collet-Eckmann rational functions. Ann. Acad. Fenn. Math. 26(1), 125-154 (2001)

23. Przytycki, F., Urbański, M.: Fractals in the plane-the ergodic theory methods. Cambridge University Press, Cambridge (2010)

24. Barański, K.: Hausdorff dimension and measures on Julia sets of some meromorphic functions. Fund. Math. 147(3), 239-260 (1995)

25. Kotus, J., Urbański, M.: Conformal, geometric and invariant measures for transcendental expanding functions. Math. Ann. 324(3), 619-656 (2002)

26. Kotus, J., Urbański, M.: Geometry and ergodic theory of non-recurrent elliptic functions. J. Anal. Math. 93, 35-102 (2004)

27. Kotus, J., Urbański, M.: The dynamics and geometry of the Fatou functions. Discrete Contin. Dyn. Syst. 13(2), 291-338 (2005)

28. Mayer, V., Urbański, M.: Gibbs and equilibrium measures for elliptic functions. Math. Z. 250(3), 915-946 (2005)

29. Urbański, M., Zdunik, A.: The finer geometry and dynamics of exponential family. Michigan Math. J. 51(2), 227-250 (2003)

30. Urbański, M., Zdunik, A.: Real analyticity of Hausdorff dimension of finer Julia sets of exponential family. Ergodic Theory Dyn. Syst. 24(1), 279-315 (2004)

31. Urbański, M., Zdunik, A.: Geometry and ergodic theory of non-hyperbolic exponential maps. Trans. Am. Math. Soc. 359(8), 3973-3997 (2007)

32. Coiculescu, I., Skorulski, B.: Thermodynamic formalism of transcendental entire maps of finite singular type. Monatsh. Math. 152(2), 105-123 (2007)

33. Coiculescu, I., Skorulski, B.: Perturbations in the Speiser class. Rocky Mt. J. Math. 37(3), 763-800 (2007)

34. Skorulski, B.: The existence of conformal measures for some transcendental meromorphic functions. Contemp. Math. 396, 169-201 (2006)

35. Kotus, J., Urbański, M.: Fractal measures and ergodic theory of transcendental meromorphic functions. In: Transcendental Dynamics and Complex Anaysis, London Mathematical Society Lecture Note Series 348, pp. 251-316. Cambridge University Press, Combridge (2008)

36. Mayer, V., Urbański, M.: Geometric thermodynamical formalism and real analyticity for meromorphic functions of finite order. Ergodic Theory Dyn. Syst 28(3), 915-946 (2008)

37. Mayer, V., Urbański, M.: Thermodynamical formalism and multifractal analysis for meromorphic functions of finite order. Mem. Am. Math. Soc. 203(954), 6+107 (2010) 
38. Mayer, V., Urbański, M.: Ergodic properties of semi-hyperbolic functions with polynomial Schwarzian derivative. Proc. Edinb. Math. Soc. 53(2), 471-502 (2010)

39. Zheng, J.H.: Dynamics of hyperbolic meromorphic functions. Discrete \& Continuous Dyn. Syst. (A) 35(5), 2273-2298 (2015)

40. Zheng, J.H.: Transfer operator and conformal measures for a class of maps having covering property. arXiv: 1303.7072

41. Zheng, J.H.: Parabolic meromorphic functions. Pac. J. Math. 250(2), 487-509 (2011)

42. Urbański, M., Zdunik, A.: The parabolic map $\frac{1}{e} e^{z}$. Indag Math. 15(3), 419-433 (2004)

43. Denker, M., Urbański, M.: Hausdorff and conformal measures on Julia sets with a rationally indifferent periodic point. J. Lond. Math. Soc. 43(1), 107-118 (1991)

44. Dudley, R. M.: Real Analysis and Probablity. Chapman and Hall, London (1989) 